\title{
39. EL GÉNERO ANDRYALA L. (COMPOSITAE, CICHORIEAE) EN LA PENÍNSULA IBÉRICA Y BALEARES: UNA NUEVA ESPECIE E HIIBRIDOS INTERESPECÍFICOS
}

\author{
Salvador TALAVERA y María TALAVERA
}

Recibido el 2 de noviembre de 2015, aceptado para su publicación el 9 de noviembre de 2015

The genus Andryala L. (Compositae, Cichorieae) in the Iberian Peninsula and Balearic Islands: a new species and interspecific hybrids

Palabras clave. Corología, sistemática, taxonomía, tipificación, novedades taxonómicas.

Key words. Chorology, Systematic, taxonomy, tipification, new records.

El género Andryala L. reúne cerca de 20 especies, con dos centros de diversidad, la Región Mediterránea y Macaronesia (archipiélagos de Madeira y Canarias), con la mayor concentración de taxones en el NW de África, sobre todo en Marruecos y Argelia.

Estudios moleculares han mostrado que el género Andryala forma un grupo monofilético junto con Schlagintweitia Griseb., Hieracium L., Hispidella Lam. y Pilosella Vaill., los cuales constituyen la subtribu Hieraciinae de la tribu Cichorieae (Asteraceae). Schlagintweitia es el género basal de la subtribu; Andryala es el género hermano de Hieracium, Hispidella y Pilosella. Hispidella y Pilosella son hermanos filogenéticos (Fehrer et al., 2009). Todos los géneros de la subtribu Hieraciinae se caracterizan por la presencia de pelos largos, escábridos o subplumosos en las hojas, excepto Andryala, en la que todos los pelos de las hojas son estrellados.

Como es frecuente en la subtribu, cuando las especies de Andryala conviven en el mismo ecosistema es habitual que se produzcan híbridos interespecíficos, sobre todo cuando uno de los parentales es $A$. integrifolia L., la especie más común en la Península Ibérica. De hecho, aunque las especies presentes en el territorio de esta flora están bien diferenciadas morfológicamente, la presencia de los híbridos dificulta bastante su identificación. En la Península Ibérica e islas Baleares se han reconocido 6 especies, una de ellas, Andryala cintrana, nueva para la ciencia, y en todas, excepto en $A$. agardhii Haens. ex DC., se han encontrado híbridos interespecíficos, algunos de ellos ya indicados por García Adá et al. (1996) y García Adá (1992).

En el presente trabajo se relacionan las especies reconocidas para el territorio de Flora iberica, y se aporta una clave para su reconocimiento. Para cada una de ellas, además, se indica el nombre correcto y principales sinónimos, su distribución, apetencias ecológicas y fenología, así como comentarios sobre su variabilidad y sobre los híbridos detectados (al final del texto); los taxones nuevos se acompañan también de una descripción de los principales caracteres taxonómicos. Se efectúan tipificaciones de buena parte de las especies e híbridos. Una selección del material estudiado se relaciona

Este trabajo ha sido financiado por el proyecto Flora iberica (CGL2012-32914) y cofinanciado con fondos FEDER 
en el Anexo, al final del texto.

Andryala L., Sp. Pl.: 808 (1753)

$=$ Rothia Schreb., Gen. Pl. 2: 531 (1791)

= Paua Caball. in Bol. Real Soc. Esp. Hist. Nat. 16: 540 (1916)

= Pietrosia Nyár. in Rev. Biol. (Bucharest)

8(3): 250 (1963)

Especie tipo: Andryala integrifolia L., Sp. Pl.: 808 (1753), designado por Green (1929: 178).

Hierbas anuales, bienales o perennes, o sufrútices, caulescentes, uni- o pluricaules, densamente pelosas, con pelos estrellados en los tallos, hojas y en el dorso de las brácteas del involucro, a veces acompañados de pelos glandulíferos en la parte superior de los tallos, pedúnculos y en el dorso de las brácteas del involucro, sobre todo en el nervio medio, a veces también en el margen de las hojas más superiores de los tallos. Tallos erectos o ascendentes, cilíndricos -estriados sobre todo en la base-, ápteros, simples o más frecuentemente ramificados en la mitad o en el tercio superior, rara vez desde la base, foliosos. Hojas de oblanceoladas a espatuladas, enteras, dentadas, lobadas o pinnatífidas, pinnatinervias, densamente pelosas por ambas caras; las basales formando rosetas, pecioladas, con el pecíolo estrecho $\mathrm{y} \pm$ largo, $\mathrm{o}$ ancho y poco diferenciado del limbo, a veces marcescentes en la floración; las caulinares similares en morfología a las basales pero generalmente más pequeñas, rara vez mayores, las más inferiores frecuentemente pecioladas, las superiores \pm sésiles o subsésiles. Capítulos solitarios o, más frecuentemente, agrupados en una inflorescencia racemiforme, paniculiforme o corimbiforme, densa o laxa, oligocéfala o policéfala, erectos antes de la antesis, \pm pedunculados, multifloros. Involucro \pm cilíndrico o campanulado en la floración, cónico o subgloboso en la fructificación, patente o reflexo después de la dispersión, con 2-5 series de brácteas internas, acompañadas de 1-2 series de brácteas suplementarias o externas, soldadas a distintas alturas en la base del involucro, más cortas y estrechas que las internas; brácteas lanceoladas, \pm rectas, planas o incurvas en la base, sobre todo las más internas, rectas o arqueadas y frecuentemente subcilíndricas las suplementarias, ventralmente glabras. Receptáculo alveolado, con los alvéolos \pm pentagonales, de margen escarioso y dentado, glabros o pelosos, con los pelos simples, unicelulares, frecuentemente mayores que los aquenios, a veces parcialmente paleáceo, con las páleas indiferenciadas de las brácteas del involucro, o de su morfología pero escariosas y glabrescentes, dispuestas en la periferia del receptáculo. Flores hermafroditas, liguladas, con 5 dientes, las externas del capítulo algo mayores que las internas. Corola vilosa en la base del limbo, amarilla, a menudo la de las flores más externas del capítulo con los dientes y el dorso del limbo \pm rosados. Anteras amarillas. Ramas estilares amarillas, a veces con el área estigmática anaranjada. Aquenios homomorfos, rara vez dimorfos, obcónicos, glabros, con 10 costillas longitudinales que alcanzan el ápice, los homomorfos generalmente provistos de una corona entera o más frecuentemente dentada y blanca que rodea a la cicatriz del estilo, en cuya parte externa se inserta el vilano, los dimorfos con los aquenios más externos del capítulos contraídos en el ápice, con el extremo de las costillas muy próximos, sin corona, carentes de vilano, de color más pálido que los internos que tienen vilano y corona. Vilano formado por una fila de pelos subplumosos en el tercio inferior y escábridos en los dos tercios superiores, o enteramente escábridos, muy débilmente soldados entre sí en la base, prontamente caedizos, blancos. $x=9$.

\section{Clave para las especies}

1. Sufrútices; hojas densamente cubiertas de pelos estrellados, que ocultan completamente la epidermis; aquenios 2-4 mm; brácteas del 
involucro reflexas después de la dispersión de los aquenios

- Hierbas anuales, bienales o perennes; hojas de laxa a densamente cubiertas de pelos estrellados, que ocultan o no totalmente la epidermis; aquenios 0,8-1,6 mm; brácteas del involucro patentes, rara vez reflejas después de la dispersión de los aquenios ..... .3

2. Al menos la mitad superior de los tallos y las brácteas del involucro con pelos glandulíferos negros; hojas de espatuladas a oblanceoladas, enteras, casi todas en la base; aquenios (3)3,5-4 x 0,7-1 mm, parduscos, con las costillas obtusas, sin corona central en el ápice.... 6. A. agardhii - Tallos y brácteas del involucro sin pelos glandulíferos; hojas de lineares a oblanceoladas, enteras, lobadas o pinnatífidas, casi todas en la base o a lo largo de los tallos; aquenios 2-2,7 x 0,4-0,6 mm, pardo-obscuros, casi negros, con las costillas agudas y blancas, con una corona central en el ápice 5. A. ragusina

3. Hojas densamente cubiertas de pelos estrellados, que ocultan completamente la epidermis; hierbas bienales o perennes, sin pelos glandulíferos; tallos ramificados desde la base, o en la mitad superior, con las ramas densamente cubiertas de hojas y de capítulos pedunculados en casi toda su longitud, formando inflorescencias racemiformes muy densas ........ 3. A. cintrana

- Hojas laxamente cubiertas de pelos estrellados, que dejan ver la epidermis al menos parcialmente; hierbas anuales, bienales o perennes, con pelos glandulíferos, al menos en el dorso de las brácteas del involucro; tallos por lo general ramificados en la mitad superior, con las ramas simples o divididas, formando inflorescencias racemiformes, paniculiformes o corimbiformes, laxas o densas, rara vez simples y monocéfalos.

4. Hierbas perennes, rara vez bienales, con rizoma vertical engrosado y a veces leñoso en el ápice; hojas caulinares medias \pm pecioladas; brácteas del involucro no encerrando flores o aquenios 1. A. integrifolia

- Hierbas anuales, con raíz axonomorfa \pm delgada; hojas caulinares medias sésiles, auriculadas o subauriculadas en la base; brácteas del involucro, casi todas o parte de ellas, encerrando flores o aquenios .5

5.Tallos por lo general divaricados en la mitad superior, formando cada rama una inflorescencia racemiforme y densa; pedúnculos casi siempre sin pelos glandulíferos o éstos muy escasos; involucro 5,5-9 x 3-10 mm, \pm cónico en la fructificación, con dos series de brácteas internas, pero solo en la serie externa cada bráctea encierra una flor o aquenio; todos los aquenios con vilano 2. A. arenaria

- Tallos ramificados en la mitad o en el tercio superior, formando cada rama una inflorescencia paniculiforme laxa; pedúnculos con numerosos pelos glandulíferos; involucro (9)12-16 x 12-16 $\mathrm{mm}, \pm$ globoso en la fructificación, con 5-6 series de brácteas internas, pero solo en las 4-5 series más externas cada bráctea encierra una flor o aquenio; aquenios más externos del capítulo sin vilano, los demás con vilano \pm desarrollado 4. A. rothia

1. Andryala integrifolia L., Sp. Pl.: 808 (1753) 三Andryala corymbosa Lam., Encycl. 1: 153 (1783), nom. superfl.

三 Andryala integrifolia var. corymbosa (Lam.) Willk. in Willk. \& Lange, Prodr. Fl. Hispan. 2: 271 (1865), nom. illeg.

$\equiv$ Rothia runcinata Roth, Catal. Bot. 1: 107 (1797), nom. illeg.

$\equiv$ Andryala runcinata (Roth) Pers., Syn. Pl.

2: 378 (1807) [348 in publ.]

$\equiv$ Rothia lanata Bubani, Fl. Pyren. 2: 80 (1899), nom. illeg.

Ind. loc.: "Habitat in Gallia, Sicilia".

Lectótipo: designado por House [(1993: 18); Herb. Burs. VI: 57 (UPS)]

= Andryala sinuata L., Sp. P1.: 808 (1753) [Ind. loc. "Habitat Montpelii, inque Sicilia “. Lectótipo: designado por House, 1998: 153; Herb. Clifford: 387, Andryala $N^{\circ} 1$ (BM- 000646869)]

三Andryala integrifolia var. sinuata (L.) L., Syst. Nat., ed. 12, 2: 525 (1767)

$\equiv$ Rothia cheiranthifolia Roth, Catal. Bot. 1: 105 (1797), nom. illeg.

= Andryala mollis Asso, Mant. Stirp. Aragon.: 175, Tab. X. Fig. 1 (1781) [Ind. loc. "Provenit en Vicor; en el monte de Herrera; circá Rodenas. Vidimus eandem plantam anno 1779. in Horto Matritensi, quam pro varietate $A$. integrifoliae habebat 
optimus praeceptor Antonio Palau". Lectótipo (designado aquí): in Asso, Mantissa Stirpium Indigenarum Aragoniae: 175, Tab. X, Fig. 1 Andryala mollis (1781)] =Andryala allochroa Hoffmanns. \& Link, Fl. Portug. 2: 154 (1825-1828) [Ind. loc. "Frecuént por tout le Royaume, le long dè chemins et sur la lisiére des champs “. Lectótipo (designado aquí): in Hoffmannsegg \& Link, Flore Portugaise 2: 154, Tab. 91 Andryala allochroa (1825-1828)]

= Andryala coronopifolia Hoffmanns. \& Link, Fl. Portug. 2: 155 (1825-1828) [Ind. loc. "Prés de Braga, sur les murs." Lectótipo (tipificado aquí): in Hoffmannsegg \& Link, Flore Portugaise 2: 155, Tab.92 Andryala coronopifolia (1825-1828)]

=Andryala dissecta Hoffmanns. \& Link, Fl. Portug. 2: 153 (1825-1828) [Ind. Loc. “” Aux lieux sablonneux en-delá du Tage près de Lisbonne, Setuval etc.”. Lectótipo: no encontrado, probablemente destruido en la Segunda Guerra Mundial].

\section{Ecología, fenología y distribución}

Ruderal y viaria, a veces dentro del matorral y bosques pastoreados, en todo tipo de substratos; 5-1600 m. II-IX(X-I). Región Mediterránea, posiblemente introducida en Canarias (Gran Canaria). Casi toda la Península Ibérica, rara en la cordillera cántabro-pirenaica y en Baleares. Esp.: A Ab Al Av B Ba Bi Bu C $\mathrm{Ca}$ CC Co CR Cs Cu Ge Gr Gu H (Hu) J (L) Le Lo Le M Ma Mu Na O Or P PM[Mn] Po S Sa Se So SS T Te To V Va (Vi) Z Za. Port.: AAl Ag BA BAl BB BL (DL) E Mi R TM.

\section{Observaciones}

Especie muy variable en el porte, morfología de las hojas y en el tipo de inflorescencia, pero con caracteres de indumento, flores y frutos muy constantes.

Las plantas de floración otoñal o invernal suelen ser débiles, y presentan hojas lineares o linear-elípticas, inflorescencias muy reducidas, a veces monocéfalas, e involucros pequeños. Por el contrario, las plantas de floración primaveral suelen ser robustas y con inflorescencias en corimbo denso, por lo que numerosos autores la identificaron como Andryala corymbosa (A. integrifolia L. s.s.), o como Andryala sinuata L. (=A. allochroa) cuando es laxo.

Plantas con hojas muy estrechas, pinnatisectas, con lóbulos lineares e inflorescencia paniculiforme laxa son poco frecuentes, y se han observado solo en Portugal (Alto Alentejo; SEV 234949), donde fueron descritas como Andryala coronopifolia Hoffmanns. \& Link de los alrededores de Braga, y como Andryala dissecta Hoffmanns. \& Link cerca de Lisboa. En Marruecos, sobre todo en sotos de Cedrus atlantica, viven plantas semejantes en todos los caracteres a Andryala integrifolia, pero con hojas basales por lo general verdes durante la floración. Estas formas se han denominado Andryala cedretorum Maire in Mém. Soc. Sci. Nat. Maroc 15: 45 (1926) [三 Andryala integrifolia subsp. cedretorum (Maire) Sauvage in Trav. Inst. Sci. Chérifien, Sér. Bot., 22: 203 (1961)].

2. Andryala arenaria (DC.) Boiss. \& Reuter, Pugill. Pl. Afr. Bor. Hispan.: 71 (1852)

$\equiv$ Andryala tenuifolia var. arenaria DC., Prodr. 7: 245 (1838) [basión.]

$\equiv$ Andryala dentata subsp. arenaria (DC.)

Nyman, Consp. Fl. Eur.: 438 (1879)

$\equiv$ Andryala rothia subsp. arenaria (DC.) Maire in Jahandiez \& Maire, Cat. Pl. Maroc: 1167 (1941)

Ind. loc.: “in arenosis maritimis circá Gibraltariam legit cl. Boissier”. Lectótipo (designado aquí): Etiqueta 1. Andryala arenaria $\mathrm{N} /$ Affinis andr. tenuifoliae Dc / sed videtur distincta / in arenosis maritimis / Gibraltar / m. Edm. Boissier 1838 (manuscrito Boissier). Etiqueta 2. andryala tenuifolia $\Upsilon$ arenaria $\mathrm{dC}$ (manuscrito A.P. de Candolle). El lectótipo 
lo componen dos plantas de unos $10 \mathrm{~cm}$ ramificadas en la parte superior, con hojas dentadas (G-DC-00319814).

= Andryala arenaria var. pinnatifida Lange ex Willk. in Willkomm \& Lange, Prodr. Fl. Hispan. 2: 272 (1865) [Ind. loc.: "in agro Madrit. ad Casa del Campo, LGE”. Lectótipo (designado aquí): J. Lange / Andryala arenaria var. pinnatifida / in agro Matritensis ad Casa de Campo 3-VI-1851 (COI-Willk.- 41885)]

\section{Ecología, fenología y distribución}

Pastizal de matorrales y bosques, en substratos generalmente arenosos; 10-800(1350) m. IV-VII. NW de África (Marruecos, Argelia y Túnez), y Península Ibérica. Frecuente en el cuadrante suroccidental de la Península, más rara en el centro y SE de España. Esp.: Al Ba Ca Cc Co CR Gu H J M Ma P Sa Se To. Port.: AAl Ag BAl BL E R.

\section{Andryala cintrana S. Talavera \& M.Talavera,} sp. nov.

Holotype: SEV248703. PORTUGAL. Estremadura. Playa de Cascais, arenales marítimos, $15 \mathrm{~m}, 38^{\circ} 43^{\prime} 42^{\prime}{ }^{\prime} \mathrm{N}-9^{\circ} 28^{\prime} 29^{\prime} \mathrm{W}$, 26.VII.2009, M. Talavera, S. Talavera \& al. (Isotype: SEV248704). Paratype: Estremadura. Cascais, playa de Guincho, 16.VI.1961, J. Malato Beliz \& J. A. Guerra (MA-273054); ibídem, a $30 \mathrm{~m} \mathrm{~N}$ del faro de guía, arenales costeros, $38^{\circ}$ $42^{\prime} 43^{\prime \prime} \mathrm{N}-9^{\circ} 29^{\prime} 4^{\prime \prime} \mathrm{W}, 18-\mathrm{VI}-2015$, D. Cuestas, C. Sánchez, M. Talavera \& S. Talavera, 164.2/15 (SEV-286489); Reserva Natural CascaisSintra. Playa de Crismina, dunas costeras, 13 $\mathrm{m}, 38^{\circ} 43^{\prime} 31^{\prime \prime} \mathrm{N}-9^{\circ} 28^{\prime} 30^{\prime \prime} \mathrm{W}, 18-\mathrm{VI}-2015$, D. Campos, C. Sánchez, M. Talavera \& S. Talavera, no 165.15 (SEV-286491).

Plant 6-20 (30) cm, usually perennial, globose or elliptical, with a dense covering of stellate hairs, 0.8-1.5 $\mathrm{mm}$ in diameter, that completely obscure the epidermis of the stems, leaves and involucral bracts. Lacking glandular hairs. Stems 3-3.5 mm in diameter at the base, erect or ascending, branched from the base or in the upper half, with numerous leaves that are close to each other, giving a dense form. Basal leaves (0.6)1.2-4.5 x (0.2)0.6-1 cm, elliptic or linear-elliptic, with the petiole short and little differentiated; stem leaves $1-3.5 \times 0.5-2 \mathrm{~cm}$, sessile, cordiform. Capitula pedunculate or subsessile in the leaf axils, united in very dense racemose synflorescences on each branch. Involucre 6-7 x 5-7 mm, subcylindrical at flowering, stellate or with the bracts reflexed after dispersal, with two series of internal bracts and three supplementary bracts at the base of the involucre; internal bracts lanceolate, acute at apex, the outermost involute and each enclosing a flower or achene, the inner bracts flat and not enclosing a flower or achene. Receptacle alveolate, with lax hairs 0.8-1.5 mm. Flowers golden yellow, the outermost with the outer side or apex purplish. Anthers 2.2-2.5 $\mathrm{mm}$, yellow. Stylar arms 0.2-0.3 mm, yellow. Achenes homomorphic, 1-1.3 x (0.35)0.4-0.5 $\mathrm{mm}$, the outermost somewhat larger than the inner, all with a pappus; pappus 4-4.5 mm, subplumose in the lower half, scabrous above, white, fragile.

Planta 6-20(30) cm, por lo general perenne, erecta, densamente cubierta de pelos estrellados de 0,8-1,5 $\mathrm{mm}$ de diámetro, que ocultan completamente la epidermis, sin pelos glandulíferos; rizoma vertical, muy engrosado en el ápice, hasta de $12 \mathrm{~mm}$ de diámetro, con numerosos restos de hojas de floraciones anteriores. Tallos de 3-3,5 mm de diámetro en la base, erectos o ascendentes, con numerosas hojas muy próximas unas de otras, ramificados desde la base o en la mitad superior, formando en conjunto una figura globosa o elíptica. Hojas basales $(0,6) 1,2-4,5 \times(0,2) 0,6-1 \mathrm{~cm}$, elípticas o linear-elípticas, con pecíolo corto y poco diferenciado, las caulinares de 1-3,5 x 0,5-2 $\mathrm{cm}$, sésiles, cordiformes, agudas. Capítulos pedunculados o subsésiles en las axilas de las 
hojas, reunidos en una inflorescencia racemosa muy densa en cada rama. Involucro 6-7 x 5-7 $\mathrm{mm}$, subcilíndrico en la floración, en forma de estrella o con las brácteas reflejas después de la dispersión, con 2 series de brácteas internas y 3 brácteas suplementarias cerca de la base del involucro, sin glándulas; brácteas internas lanceoladas, agudas, las más externas de las dos series involutas, encerrando una flor o un aquenio cada una, las más internas planas. Receptáculo alveolado, laxamente peloso, con pelos de 0,8-1,5 mm. Flores amarillas, las más externas con el dorso o el ápice purpúreo. Anteras 2,2-2,5 mm, amarillas. Ramas estilares 0,2-0,3 mm, amarillas. Aquenios homomorfos, de $1-1,3$ x $(0,35) 0,4-0,5 \mathrm{~mm}$, los externos algo mayores que los internos, todos con vilano. Vilano 4-4,5 mm, subplumoso en la mitad inferior, escábrido en la superior, blanco, caedizo.

\section{Ecología, fenología y distribución}

Coastal sandy pastures; 10-30 m. VI-VIII. Centre-West of Portugal, Natural Park CascaisSintra. CW Portugal. Port.: E.

\section{Observaciones}

$A$. cintrana convive con $A$. arenaria y $A$. integrifolia, con las que a menudo se hibrida.

4. Andryala rothia Pers., Syn. Pl. 2: 378 (1807) $\equiv$ Voigtia tomentosa Roth in Bot. Mag. (Römer \& Usteri) 10: 17 (1790), syn. subst., non Andryala tomentosa Chaix (1785)

$\equiv$ Rothia andryaloides Gaert., Fruct. Sem. P1. 2: 371 (1791), nom. illeg.

$\equiv$ Andryala rosea Steud., Nom. Bot., ed. 2, 2: 779 (1841), nom. illeg.

Ind. loc.: "Semina hujus plantae inter Passulos majores legi, quae hoc anno terrae steriliori horti mei commisi. Horis antemeridianis efforescit" ["habitat in Hispania", según Roth, Catal. Bot. 1: 105 (1797)]

Lectótipo: designado por García Adá et al. in Candollea 51: 377 (1996) [(B-Will No. 14770)].
= Andryala laxiflora DC., Prodr. 7: 246 (1838) [Ind. loc.: "in Mauritaniâ circà Tanger legit cl. Salzmann et circa Malagam cl. Boissier. Rothia laxiflora Salzm.! pl. exs. 1825". Lectótipo: En el herbario de A. P. de Candolle no se ha encontrado el material de Málaga recolectado por Boissier, y el de Tánger de la exsiccata de Salzmann, al menos los pliegos estudiados de dicha exsiccata, contiene material heterogéneo. Un pliego con material de Andryala integrifolia (MPU-001527), otro, con tres plantas de Andryala laxiflora, pero con capítulos mas pequeños de lo habitual en Andryala rothia (MPU- 001367) y 7 pliegos de la exsicata con materiales que se podrían identificar como de origen híbrido de $A$. integrifolia con $A$. rothia (MPU-001529, 001528, 001526, 001525, 001524, 001366, \& MPU-001365), es decir lo que se ha denominado Andryala x caballeroi Font Quer. Si el material de Málaga de $A$. laxiflora indicado por A. P. de Candolle se ha destruido y hay que elegir el lectótipo del material de Tánger recolectado por Salzmann, probablemente Andryala laxiflora $\mathrm{DC}$ habría que considerarla como de origen híbrido de $A$. integrifolia con $A$. rothia]

$\equiv$ Andryala sinuata subsp. laxiflora (DC.) Nyman, Consp. Fl. Eur.: 438 (1979)

$=$ Andryala arenaria subsp. mariana Rivas Goday \& Bellot in Bol. Soc. Esp. Hist. Nat. 40: 64 (1942) [Ind. loc.: “ $\mathrm{Hab}$. in rupestribus apricis, loco dicto Valdeazores, circa Despeñaperros, Montium Marianorum: 650 alt. ubi d. 12 junii 1941, invenimus". Lectótipo (designado aquí): Etiqueta 1. Herbario de la Facultad de Farmacia de Madrid / Plantae Regionis Centralis Hispaniae ex S. Rivas Goday et / F. Bellot, lectae / ANDRYALAARENARIA (DC.) B et R. s. sp. MARIANA / in collibus apricis loco dicto Valdeazores / 
(Despeñaperros) Montium Marianorum / 12-VI-41 (MAF-09056). Etiqueta 2. Holotypus!/Herbario Facultad FarmaciaMadrid (MAF) / Andryala arenaria subsp. mariana Rivas Goday \& Bellot / (A. laxiflora DC) / MAF 9056 / J. Pizarrro Det 11. Feb. 2013 / Bol. Soc. Esp. Hist. Nat. 40: 64 (1942)". El tipo lo constituyen dos plantas de unos $25 \mathrm{~cm}$, ramificadas en la base, con los capítulos dispuestos en corimbos laxos]

\section{Ecología, fenología y distribución}

Pastizales de terófitos en substratos arenosos, pizarrosos o margosos, a veces también en cultivos de almendros, vides, etc.; 10-850 m. V-VII. W de la Región Mediterránea (Península Ibérica, Marruecos y Argelia); posiblemente adventicia en Canarias (Tenerife). C y S de la Península Ibérica. Esp.: Av Ba Ca Cc Co CR H J M Ma Sa Se To. Port.: Ag BA BAl BB TM.

\section{Observaciones}

A pesar de los caracteres tan distintivos que tiene $A$. rothia, la hibridación con $A$. integrifolia o $A$. arenaria complica a veces la identificación de esta especie.

5. Andryala ragusina L., Sp. P1., ed. 2: 1136 (1763)

Ind. loc.: "Habitat in insulis Archipelagi"

Lectótipo: designado por House, 1998: 351 [(Her. Linn. No956.4 (LINN)]

\section{Ecología, fenología y distribución}

Claros de matorral, en calizas, dolomías, margas, esquistos, e incluso en cascajares de ríos o arenales marítimos, a veces en viñedos u otros cultivos de plantas leñosas; 10-2500 m. (I)III-VIII(IX). Península Ibérica, Baleares y S de Francia. Casi todas las provincias de la Península, muy rara o ausente en la fachada atlántica. Esp.: A Ab Al Av B Ba Bu Ca Cc Cs $\mathrm{Cu}$ Ge Gr Gu Hu J L Le Lo Lu M Ma Mu Na
Or P PM[Ib] S Sg So T Te To V Va Z Za. Port.: AAl BB TM.

\section{Observaciones}

Especie muy variable en la mayoría de los caracteres, pero fácilmente reconocible por la ausencia de pelos glandulíferos en todos sus órganos vegetativos, como $A$. cintrana. En la Península Ibérica existen dos pautas de variación si atendemos al tamaño del involucro.

Las plantas con capítulos grandes o medianos, que aparecen por toda el área de distribución de la especie, preferentemente en el centro de España, se identifican con $A$. ragusina L. s.s. [ = Andryala lyrata Pourr. in Hist. Mem. Acad. Roy. Sci. Toulouse 3: 307 (1788). Ind. loc. "cette superbe plante est très-commune sur les bords des petites rivieres des hautes Corbieres, notamment à Pader, St. Paul de Fenouilhedes \& c". Tipo: no encontrado; 三 Andryala ragusina var. lyrata (Pourr.) Willk. in Willk. \& Lange, Prodr. Fl. Hispan. 2: 271 (1865); = Andryala macrocephala Boiss. ex DC., Prodr. 7: 244 (1838). Ind. loc. "ad torrentes litorales regni Granatensis legit $\mathrm{cl}$. E. Boissier". Lectótipo: designado por Burdet \& al. (1983: 757) (G-DC)]; 三 Andryala lyrata var. macrocephala (Boiss. ex DC.) Boiss., Voy. Bot. Midi Espagne 2: 393 (1839) E Andryala ragusina subsp. macrocephala (Boiss. ex DC.) Nyman, Consp. Fl. Eur.: 438 (1879; = Crepis incana Lapeyr., Hist. Pl. Pyrénées: 483 (1813), non Sm. (1813) EAndryala incana DC. in Lam. \& DC., Fl. Franç., ed. 3, 5: 445 (1815), nom. nov. Ind. loc. "Dans les sables de la rivière de Sin et de Plan". Tipo: no estudiado; $\equiv$ Andryala lyrata var. incana (DC.) DC., Prodr. 7: 244 (1838)]. Estas plantas suelen tener también las hojas caulinares grandes y anchas, pedúnculos robustos, y los órganos florales presentan las medidas mayores de la especie.

Las plantas con capítulos pequeños que se distribuyen por la mitad oriental de la Península Ibérica, principalmente por la costa del Mediterráneo, se identifican con Andryala 
ragusina var. ramosissima Boiss. ex DC., Prodr. 7: 244 (1838) [Ind. loc. "in collibus siccis et calcareis maritimis regni Granatensis usque ad 5000 ped. altit. legit cl. Boissier". Lectótipo: designado por Burdet \& al. 1983: 758 (G-DC); 三 Andryala ramosissima (Boiss. ex DC.) Boiss., Elenchus Pl. Nov.: 63 (1838) $\equiv$ Andryala lyrata var. ramosissima (Boiss. ex DC.) Boiss., Voy. Bot. Midi Espagne 2: 393 (1839); = Andryala lyrata f. sordida Font Quer in Bol. Soc. Esp. Hist. Nat. 20: 175 (1920). Ind. loc. "Ibiza: Cala de Molins, Gros, 24-V-1918". Lectótipo: designado por Roselló \& Sáez, 2000: 24 (BC-36082)], o con Andryala ragusina var. minor (Lange) Willk. in Willk \& Lange, Prodr. Fl. Hispan. 2: 271 (1865) [三 Andryala lyrata var. minor Lange in Vidensk. Meddel. Dansk Naturhist. Foren. Kjøbenhavn: 103 (1861), basión.; Ind. loc. "in arenosis secùs fluminum ripas agri Narbonensis et Ruscinonensis". Tipo: no estudiado; $\equiv$ Andryala ragusina var. lyrata DC, Prodr. 7: 244 (1838), non Adryala lyrata Pourret].

Andryala ragusina var. ramosissima vive en las dolomías cristalinas de Sierra Tejeda y en la Serranía de Ronda, y se caracteriza por tener la mayoría de las hojas en la base, cortas y estrechas, y las caulinares casi bracteiformes [Esp. Gr Ma]. Por el contrario, las plantas de la var. minor se caracterizan por tener las hojas de la base generalmente largas y anchas, y las caulinares casi del tamaño de las basales. Estas plantas viven preferentemente en el levante, desde Granada hasta Gerona [Esp.: A Al B Cs Ge Gr Mu T V], por lo general en substratos arenosos cercanos al mar, pero también aparecen muy esporádicamente en diversas provincias del interior [Esp.: $\mathrm{Cu} \mathrm{Gr} \mathrm{Hu} \mathrm{J}$ Lo M S Sg T Te V], lo que nos impide reconocerla como subespecie.

La hibridación interespecífica es muy rara en esta especie ya que solo se han observado individuos de $A$. ragusina con algunos caracteres de $A$. integrifolia en el centro de España.
6. A. agardhii Haens. ex DC., Prodr. 7: 244 (1838)

$\equiv$ Pietrosia agardhii (Haens. ex DC.) Sennikov in Komarovia 1: 78 (1999)

Ind. loc.: "in summis Sierra Tejeda frequent, et in Sierra Nevada Hisp. Granat. rara".

Lectótipo: designado por Burdet \& al. 1983: 575 (G-DC).

\section{Ecología, fenología y distribución}

Grietas de rocas y pedregales calcáreos; 1220-2000 m. VI-VII(VIII). Península Ibérica y Marruecos. SE de España. Esp.: Ab Al Gr J $\mathrm{Ma} \mathrm{Mu}$.

\section{HÍBRIDOS DETECTADOS EN EL TERRITORIO}

\section{Andryala integrifolia L. x Andryala arenaria} (DC.) Boiss. \& Reuter

$=$ Andryala $\times$ faurei Maire in Bull. Soc. Hist. Nat. Afrique N 17: 121 (1926)

$\equiv \times$ Andryala faurei Maire in Faure, Bull. Soc. Hist. Nat. Afrique N 14: 263 (1923), nomen nudum.

Ind. loc.: "Hab. inter parentes in agro oranensi, prope $<<$ Les Lauriers-Rose $>>$ ubi leg. A. FAURE maio et junio florentem".

Lectótipo (designado aquí): Etiqueta 1: "Herbier Université de Montpellier, type". Etiqueta 2: "Herbier A. Faure / Plantes de l'Algérie /Andryala arenaria $\mathrm{x}$ integrifolia / Les Lauriers-Roses (Oran) / Lieux incultes près de la Gare / Entre les parents présumés / 29 mai 1921 A. Faure" (MPU 001870). El tipo lo constituye una sola planta de ca. $60 \mathrm{~cm}$, con las hojas inferiores del tallo pecioladas y la inflorecencia en corimbo denso, como en $A$. integrifolia, pero con capítulos de involucro pequeño, como los de $A$. arenaria.

$=$ Andryala $\times$ humilis Pau in Font Quer, Iter Marocc. 1927 (in schaed.): $n^{0} 728$ (1928), pro especie. Ind. loc.: "Hab. in umbrosis 
Yebel Dahar, pr. Axdir (Beni Urriaguel), $300 \mathrm{~m}$. alt. solo calc.; 9 majii". Lectótipo (designado aquí): BC-36140; GDA-2571 isótipo. El material tipo lo constituyen dos plantas, una de $10 \mathrm{~cm}$ y otra de $20 \mathrm{~cm}$, de las que se elige la mayor como lectótipo, una planta con la inflorescencia en corimbo laxo y las hojas basales pinnatífidas.

Este híbrido es raro en Argelia y Marruecos, pues $A$. arenaria escasea en el NW de África, pero es relativamente frecuente en la Península Ibérica [Esp.: Ca Co Cu H M Ma To. Port.: BL E R]. Las plantas suelen ser perennizantes, como A. integrifolia, con hojas caulinares y tamaño del involucro parecidos a los de $A$. arenaria. Unas veces tienen inflorescencias densas y corimbiformes, como en $A$. integrifolia ( A. $\times$ faurei s.s.), y otras laxas y racemiformes o bien paniculiformes, como en $A$. arenaria ( $A$. $\times$ humilis s.s.).

\section{Andryala integrifolia L. x Andryala rothia} Pers.

$=$ Andryala $\times$ caballeroi Font Quer in Cavallinesia 7: 83 (1935) [pro hibrido de A. integrifolia L. con $A$. arenaria sensu Font Quer]

Ind. loc.: "Hab. in Imp. Maroccano, pr. Zinat, ditione Tingitana, inter parentes, ubi d. 28 maji /050 legi.".

Lectótipo: no encontrado. Aunque no se ha podido estudiar el tipo, existe un pliego recolectado por Font Quer en Zinat, el 28-V-1930 (BC-934633), e identificado por él como “Andryala arenaria, pater. Hybr". Esta planta realmente pertenece a $A$. rothia, luego $A$. arenaria deber ser cambiado por $A$. rothia.

= Andryala integrifolia var. vidali $\mathrm{Pau}$ in Asoc. Española Progr. Ci.: 151 (1928), pro variedad hibrida [Ind. loc.: "Dar Riffien". Lectótipo (designado aquí): Etiqueta 1. Andryala rothia Pers. / var. vidali Pau / A. laxiflora x integrifolia/junio 1925 /
Marruecos Vidal y Aterido. Etiqueta 2. Andryala vidali Pau $/=$ A. laxiflora $\mathrm{x} \mathrm{A}$. angustifolia/ Dar Riffien / A. Aterido junio 1926 (MA 139360)].

$=$ Andryala $\times$ dichroa Maire in Bull. Soc. Hist. Nat. Afrique N 28: 364 (1937) [Ind. loc.: "Alger, né des semences d'un pied d'Andryala laxiflora Salzm. élévé d)akènes récoltés à Jemmapes, au milieu des A. integrifolia spontanés». Lectótipo (designado aquí): Etiqueta 1. Herbier Université de Montpellier (MPU-004363). Etiqueta 2. Herbier Dr. René Maire .Etiqueta 3. TYPE. AUCT. T. MADAULE, M. DURAND \& C. LOUP. Etiqueta 4. SOCIÉTÉ CÉNOMANE D'EXSICCATA No 3151 / x Andryala dichoroa Maire / = A. integrifolia L. x laxiflora Salzm. / ALGER: issu de semences d'A. Laxiflora, cultivé au milieu / des A. integrifolia / 6 Juin 1936 Dr. R. Maire]

Este híbrido, frecuente en Argelia y Marruecos, aparece esporádicamente en la Península Ibérica [Esp.: Ca Cc Co CR J H M. Port.: TM]. Se reconoce con facilidad por tener los capítulos largamente pedunculados, dispuestos inflorescencias laxas y racemiformes que no se parecen a las de los padres, pero suelen ser perennes, con las hojas caulinares inferiores estrechas y pecioladas, como en $A$. integrifolia, y el involucro presenta más de una serie de brácteas internas que encierran flores o aquenios, como en $A$. rothia.

Andryala integrifolia L. x Andryala ragusina $\mathrm{L}$.

$=$ Andryala $\times$ brievaensis García Adá in Acta Bot. Malacitana 17: 259 (1992).

Ind. loc.: "SEGOVIA: Brieva, 30TVL1244, $1040 \mathrm{~m}$, tomillar pedregoso sobre calizas contiguas a terrenos silíceos, 21-VII-1989, leg. R. García Adá y G. López, 6546 RG.

Holotypus: MA 503134; Isotypus: MA 
503135. Las plantas de Brieva son del todo semejantes a $A$. ragusina, con el receptáculo peloso y los aquenios estériles. Es posible que Andryala x brievaensis no tenga origen híbrido, ya que el receptáculo peloso o glabro se da indistintamente en muchas poblaciones de $A$. ragusina, y la esterilidad de las plantas está relacionada con la autoincompatibilidad, que origina aquenios estériles en plantas solitarias o un poco alejadas de otras coespecíficas.

= Andryala mollis var. burgalensis Sennen \& Pau ex Pau in Bull. Géogr. Bot. 21: 151 (1911), pro var. hybr. [Ind. loc.: "Hab. Pancorbo, friches (Elias)". Lectótipo (designado aquí): in Sennen Pl. d'Espagne 1907 No468 (1907) (MA-1319178)]

$\equiv$ Andryala mollis var. burgalensis Sennen \& Pau in Sennen Pl. d' Espagne 1907 N468 (1907), nomen nudum

Híbrido muy raro, observado solo en el centro de España [Esp.: $\mathrm{Bu} \mathrm{Sg} \mathrm{Va].} \mathrm{Los}$ híbridos de Burgos y Valladolid son perennes, sufruticosos, densamente cubiertos de pelos estrellados en los tallos, hojas y pedúnculos, parecidos a $A$. ragusina, pero los capítulos se disponen al final de los tallos formando inflorescencias corimbiformes y las brácteas del involucro presentan numerosos pelos glandulíferos, como en $A$. integrifolia.

Andryala arenaria (DC) Boiss. \& Reuter x Andryala rothia Pers.

Andryala $\times$ toletana Talavera \& M. Talavera, hybr. nov.

Holotype: ESPAÑA. Toledo. Quinta de Mora, Los Yébenes, 3-VII-1986, F. Gómez (MA-380430). Paratype: Jaén. Santa Elena, Despeñaperros, 8-VI.1924, P. Font Quer \& E. Gros (BC-916138).

Plants 6-10 cm and with the involvucre 7-9 x $9-10 \mathrm{~mm}$, as in Andryala arenaria, but with the peduncles and involucral bracts densely covered with glandular hairs, as in A. rothia.

Plantas anuales de $6-10 \mathrm{~cm}$ de altura, con involucro de 7-8 x 9-10 mm, como los de $A$. arenaria (DC.) Boiss. \& Reuter, y con los pedúnculos y brácteas internas del involucro densamente cubiertos de pelos glandulíferos, como en $A$. rothia Pers.

Andryala cintrana Talavera \& M. Talavera $\mathrm{x}$ Andryala integrifolia $\mathrm{L}$.

Andryala $x$ estremadurensis Talavera \& M. Talavera, hybr. nov.

Holotype: PORTUGAL. Estremadura. Cascais, Cabo Raso, 28 42'11'N-9'28'34'W, arenal costero, 12-V-2010, J. Calvo no JC588 (MA-807100).

Robust plants with the lower leaves 9-12 $x$ 1.5-2 cm, petiolate, laxly dentate, with a yellowish-brown indumentum of stellate hairs of 0.3-0.7 mm in dimater, as in Andryala integrifolia L.; upper leaves oval-lanceolate. sessile, auriculate, with a dense, whitish indumentum, as in A. cintrana S. Talavera \& M. Talavera. Capitula solitary at the ends of the branches - corymbose synflorescences in A. integrifolia, racemose synflorescences in A. cintrana-. lacking glandular hairs on the involucre and with the flowers golden yellow, as in A. cintrana.

Plantas robustas, con hojas inferiores del tallo de 9-12 x 1,5-2 cm, pecioladas, laxamente dentadas, con indumento pardusco de pelos estrellados de 0,3-0,7 $\mathrm{mm}$, como en Andryala integrifolia L., las superiores del tallo oval-lanceoladas, sésiles, auriculadas, con indumento blanquecino muy denso, como en Andryala cintrana Talavera \& M. Talavera. Capítulos solitarios al final de las ramas, sin pelos glandulíferos en el involucro y flores de un amarillo-dorado.

Andryala cintrana Talavera \& M. Talavera $\mathrm{x}$ Andryala arenaria (DC.) Boiss. \& Reuter 
Andryala $x$ ficalheana Daveau in Bol. Soc. Brot. 1: 42 (1883), status novo. 1879 ".

Ind. loc.: "Ile Berlenga (D.), juillet et aout

Lectótipo: no encontrado.

Although it has not been possible to study the type, we have seen a herbarium sheet with specimens collected by Daveau on the Ilhe Berlenga some years after the description of Andryala ficalheana Daveau. These comprise some individuals with hairs on the bracts of the involucre that we identify as A. arenaria, and others that are identical with the plants described by Daveau. After studying abundant material, not only from the Islas Berlengas but also from other localities in Estremadura, we consider that Andryala ficalheana Daveau may have a hybrid origin derived from Andryala cintrana y A. arenaria. Such individuals are small, with habit and indumentum similar to A. cintrana, but with involucral bracts that present numerous black glandular hairs, 1-1.5 $m m$, similar to those of $A$. arenaria.

Aunque no se ha estudiado el tipo, existe una exsiccata de Andryala ficalheana Daveau de la isla Berlenga, que fue distribuida por $\mathrm{Ch}$. Magnier con el número 1735. El material que contiene fue recolectado por Daveau delante del faro de guía en la isla Berlenga, en agosto de 1885. El material del pliego que hemos estudiado de dicha exsiccata (MA-139297) es heterogéneo, y contiene plantas de 10-20 cm, unas ramificadas en el tercio superior, con pelos glandulíferos negros de 1-1,5 mm en el dorso de las brácteas internas del involucro, que se podrían identificar con Andryala ficalheana Daveau (1883); otras están ramificadas desde la base, y tienen en el involucro pelos glandulíferos amarillentos de 0,4-0,6 mm, como en Andryala arenaria subsp. parvipila Franco, Nova Fl. Portugal 2: 574 (1984), descrita de Almeirim, cerca de Santarém (Ribatejo). Después de estudiar material abundante, no solo de la isla Berlenga, sino también de la costa continental de Estremadura, llegamos a la conclusión que A. ficalheana podría haberse originado de la hibridación de $A$. cintrana con $A$. arenaria, donde comúnmente conviven en los arenales costeros continentales, por lo que hemos propuesto el cambio de estado taxonómico.

\section{ANEXO}

\section{Material estudiado (selección)}

\section{A. integrifolia}

ESPAÑA. Alicante. Gata de Gorgos (VAL-8380). La Torre de les Mascanes (VAL-221063). Alcoy (VAL-221064). Albacete. Munera (VAL-133094). Bienservida (MA-59181). Almería. Rago (MA-139149). Sierra de Bacares (MA-139148). Ávila. Becedillas (SALA-125665). Grandes (SALA-125761). Mombeltrán (VAL-101880). Barcelona. S. Bri del Llobregal (BC-36134). Ametlla del Valles (612503). Riera de Vallvidrera ( MA-139138). Tibidabo (MA-139139). Badajoz. Mengabril (MA-459453). Pr. Badajoz (MA-139208). Salvatierra (MA-803775). Bilbao. Ubernaga (MA-139179). Burgos. Castillo de la Vega (SALA-150014). Aranda del Duero (MA-139134). Frías (MA-437637). Cáceres. Hevás (SALA-99787). Puerto de Hoyos (SALA-114596). Puerto del Robledillo (SALA-114595). Cádiz. Jimena de la Frontera (MGC-19436). Algodonales (MGC-9164). Chiclana de la Frontera (MGC-32702). Castellón. Caudiel (VAL-20470). La Pobla Tornesa (VAL-215877). Onda (VAL-174758). Rosell (VAL-20079). Ciudad Real. Daimiel (MA-551767). Puebla de Don Rodrigo (MA-615341). Puerto Llano, Puerto de la Mestanza (SALA-87425). Córdoba. Pedroches. Belalcázar (FUCO-04664). Cuenca. Barchín del Hoyo (VAL-152469). Villalba de la Sierra (VAL-158720). Maviana (VAL_158606). Gerona. Santa Coloma de Farnés (BC-879523). Cadaqués (MA-139209). El Gironés (MA-793688). La Selva (MA-783561). Granada. Alhama de Granada (MGC-58640). Almuñécar (MA-139150). Castril (MA-238517). Guadalajara. Cincovillas (MA-351530). Ventosa (MA-529717). Huelva. Escacena del Campo (MGC-48460). Islas Baleares. Menorca. Menorca, Prat de Tirant (MA-444178). Jaén. Los Villares (SALA-91276). Santiago de la Espada (VAL-151039). Cabeza Gorda 
(VAL-151041). La Coruña. Playa de Corrubedo (SALA-3613). Playa de Lago (SANT-21899). Santiago de Compostela (SANT-27571). León. Carucedo (SALA-148278). Embalse de Peñarrubia (VAL-133097). Villomar (SEV-65190). Logroño. Cerca de Logroño, orilla del Ebro (MA-139232). Ventrosa (MA-139191). Logroño MA-139193). Lugo. Monforte (SANT-23174). Seoane do Caurel (SANT-15801; SALA-115684). Madrid. Villamanta (FUCO-04665). Madrid, Ciudad Universitaria (FUCO-04083). Cadalso de los Vidrios (MA-114619). Málaga. El Humilladero (MGC-64264). Venta del Túnez (MGC-1415). Ojén, Sierra Blanca (MGC-11890). Murcia. Moratalla (MA-491046). Navarra. Torralba del Río (MA-500053). Fustiñane (MA-704603). Mendavia (704604). Orense. Carballeda (SANT-17115). Sobradelo (SANT-17073). Lobios (SAT-64769). Oviedo. Trubis (FUCO-04660). Proaza (FUCO-17122). Luarca (FUCO-14977). Palencia. San Martín de los Herreros (SALA-510). Villabellaco (MA-542371). Cervera de Pisuerga (BC-36119). Pontevedra. Vigo (FUCO-20045) Tomiño Sobrada (SANT-23163). Salvaterra de Miño (SANT-23161). Salamanca. Linares de Río Frío (FUCO-17084). San Esteban de la Sierra (SALA-5574). La Alberca (SALA-143399). Santander. Merón (MA-599553). Liencres (Ma-683904). Polaciones (MA-683903). Sevilla. El Madroño (MGC-52179). Burguillo (MGC-32682). Dos Hermanas (SALA-99152). Segovia. Martín Muñoz de la Posada (FUCO-16277). Villovega de Pirón (SANT-58186). Santiuce de Pirón (SANT-58187). Soria. Cauredondo (VAL-103763). Berianga (VAL-53760). San Sebastián. Bergara (MA-704605). Tarragona. Basses de la Foia (MA-627029). La Senia (MA-792716). Teruel. Noguera de Albarracín (VAL-197472). Ródenas (VAL-197337). Tramanastilla (VAL-160619). Toledo. Nacimiento del río Estena (MA-448155). Valencia. Simat de la Valdigna (VAL-182917). Oliva (VAL-200316). Safor (VAL-200313). Valladolid. Renedo de Esgueva (SALA-113046). Encinas de Esgueva (SALA-37746). Pozal de Gallinas (SALA-145502). Zamora. Cubo del Vino (SALA-3320). Micereces de Tera (SALA-145569). Salce (SALA-144349). Zaragoza. Calatayud (MA-139136). Morata de Jalón (VAL-209715. Sierra de Bicort (VAL-1606219). Encinacorva (VAL-83806).

PORTUGAL。A lgarve. La g oâ o (MA-420213). Vila do Bispo (MA-273047). Serra de Monchique (MA-273034). Alto Alentejo. Serra do Sâo Mamede (VAL-212031). Serra do Roxo (MA-273052). Montemor o Novo (MA-974670). Beira Alta. Manteigas (SAT-26879). Serra da Estrela (MA-273050). Mortagua (MA-169205). Baixo Alentejo. Barrancos (MA-273043). Beja (MA-777576). Beira Baixa. Covilha (MA-139097). Beira Litoral. Coimbra (SANT-26876). Miranda do Corvo (MA-822269). Penacova (MA-385017). Estremadura. Cabo de Rocha (VAL-211883). Sintra (SANT-8621). Sines (MA-414993). Península de Troia (MA-273049). Minho. Viana do Castelo (VAL-204019). Entre Torras de Douro y Covidos (SEV-234987). Ribatejo. Santaren, Sierra de Aire (MA-809901). Minde (VAL-212030). Martinchel (VAL-212032). Tras-os-Montes. Braganza (VAL-204223). Ernelo, P.N. do Alvâo (HVR-6941). Vimioso (VAL-203434).

\section{A. arenaria}

ESPAÑA. Almería. Cabo de Gata (MA-157873). Entre Abrucena y las Rozas (SEV-138193). Badajoz. Herrera del Duque, finca de Las Navas (MA-202383b). Los Santos de Maimona (MA-803719). Cádiz. Chiclana de la Frontera (SALA-65965). Bornos (MA-438296). Arcos de la Frontera (VAL-70413). Cáceres. Sierra de Gata, Los Genoveses (MA-137274). Plasencia (SALA-57211). Navalmoral de la Mata (SALA-66691). Córdoba. Villaviciosa de Córdoba (MA-748983). La Jarosa (MA-352034). Guanalnuño (SALA-87428). Ciudad Real. Puerto Llano (MA-332723). El Palancar (MA-782444). Villarrubia de los Ojos (MA-139123) Sierra de la Alhambra (MA-139122). Gudalajara. Tamajón (VAL-184428). Huelva. Cerro del Andévalo (MA-717938). Moguer, Mazagón (SALA-91291). Berrocal (MA-139287). Jaén. Bailén (SALA-48783). Andújar, Lugar Nuevo (MA-169209). Andújar, Morquilla (MA-461809). Madrid. El Pardo (VAL-142334). Colmenarejo (SANT-66086). Manzanares (MA-442465). Málaga. Ronda, El Duende (MA-139275). Marbella (VAL-96694). Sierra de Aguas (MA-442056a). Palencia. Torrao (SALA_57213). Salamanca. Valdelágene (SALA-12158). Sevilla. Gerena (MGC-88459). Puebla del Río, Venta del Cruce (MA-311880). Toledo. Talavera de la Reina (MA-352015). Navalcón (SALA-71398).

PORTUGAL. Algarve. Cabo de San Vicente (SANT-20359). Tavira (MA-273048). Alto Alentejo. Portalegre, Serra do Pico (MA-273051). Serra 
d'Ossa (MA-273045). Baixo Alentejo. Odemira, Cabo Sardâo (MA-273036). Sines (SEV-284787). Lagoa de Melides (MA-570392). Beja, Almodovar (MA-777572). Doro Litoral. Coimbra, Penacova (MA-384996). Estremadura. Colares, playa de Almocágenes (MA-138299). Cado de Roca (SANT-26877). Lagoa de Albufeira (SEV-284839). Sesimbra (MA-479165). Ribatejo. (MA-865824; VAL-211330). Castelo do Bode (SANT-26876).

\section{A. cintrana}

PORTUGAL. Estremadura. Playa de Cascais, arenales marítimos, $15 \mathrm{~m}, 38^{\circ} 43^{\prime} 42^{\prime \prime} \mathrm{N}$ 9²8'29'W, 26.VII.2009, M. Talavera, S. Talavera \& al. (Isotype: SEV248704). Cascais, playa de Guincho, 16.VI.1961, J. Malato Beliz \& J. A. Guerra (MA-273054); ibídem, a $30 \mathrm{~m} \mathrm{~N}$ del faro de guía, arenales costeros, $38^{\circ} 42^{\prime} 43^{\prime \prime} \mathrm{N}-9^{\circ}$ 29'4' 'W, 18-VI-2015, D. Cuestas, C. Sánchez, M. Talavera \& S. Talavera, 164.2/15 (SEV-286489). Reserva Natural Cascais-Sintra. Playa de Crismina, dunas costeras, $13 \mathrm{~m}, 38^{\circ} 43^{\prime} 31^{\prime \prime} \mathrm{N}-9^{\circ} 28^{\prime} 30^{\prime \prime} \mathrm{W}$, 18-VI-2015, D. Campos, C. Sánchez, M. Talavera \& S. Talavera, $n^{\circ} 165.15$ (SEV-286491).

\section{A. rothia}

ESPAÑA. Ávila. Poyales del Hoyo (SALA-44879). De Casavieja a Mijares (MA-444719). Badajoz. Herrera del Duque, finca de las Navas (MA-202383; SEV-32574). Fuente Labrada de los Montes (MA-466088). Cáceres. Magangorda (MA-257667). Deleitosa (MA-341308). Alcántara. (MA-718804) Cádiz. Sierra de la Plata (BC-876435). Córdoba. Almodóvar del Río (MA-749469). Cerca de Córdoba (BC-95014). EL Cabril (MA-784926). Ciudad Real. Despeñaperros (MGC-4365; VAL-333100). Almodóvar del Campo, La Garganta (MA-596824). San Lorenzo de Calatrava (MA-783579). Huelva. Entre Villanueva de los Castillejos y Sanlúcar del Guadiana SEV-42105). Colinas cerca de Huelva (MA-239288). Paimogo (MA-139286). Valverde del Camino (MA-444786). Jaén. Entre Bailén y Andújar (MA-139316). Puerto de Despeñaperros (MA-182139). Aldeaquemada (SEV-235047); (SANT-61651); (MA-248779). Madrid. Villa del Prado (MA-566118). Cadalso de los Vidrios (MA-566055). Entre Almorox y Cenicientos (MA-366430). Málaga. Sierra Parda de Tolox (MA-139317). Pantano del Chorro (SALA-62357). Ronda (MA-139315). De Gaucín a Casares
(MA-139314). Monda (MGC-65841). Salamanca. Villoseco de Gomitos (MA-444631). Aldeadávila (MA-456635); (MGC-23286). La Fregeneda (MA-224622). Sevilla. Gerena (MA-789475). Lora del Río (MGC-62523). Guadalcanal (MA-139313). Real de la Jara (MA-139278). Castillos de las Guardas (MA-139312). Toledo. La Herrería (MA-351538). Lillo (MA-139271a). Entre Arenas de San Pedro y Talavera de la Reina (SEV-63734). Lucillas (VAL-212610). Corchuela (SALA-71227).

PORTUGAL. Algarve. Castro Marín, Odeleinte (HVR-7401). Cachopo (MA-139298). Baixo Alentejo. Entre Évora y Redondo (SANT-26881). Beira Alta. Guarda (MA-839095). Barca d' Alva (MA-157039). Guarda, Figueira de Castelo Rodrigo (MA-939095). Sant Joâo de Sequeira (HVR-11975). Beira Baixa. Monfortinho (MA-151136). Tras os Montes. Penedo Durao (MA-592151). Bemposta (MA-806785). Freixo de Espada á Cinta (HVR-5399). Entre la estacào C.F. do Tua e a Alegría (HVR-3309).

\section{A. ragusina}

ESPAÑA. Albacete. Villa de Ves (MA-373297). Caudete (MA-429149). Peñarrubia (SALA-26688). Almería. Antás (MA-741808). Cabo de Gata (MA-648166). Ulula (MA-441682). El Almerinar (MA-352029). Sierra de Gador, Gabar (GDA-53873). Alicante. Castells de Castell (MA-586701). Sierra Mariola (MA-369205). Playa de El Altet (MA-805725). Ávila. Grandes (SALA-125762). Río Adaja (MA-313299). Sierra de la Palomera (MA-858605). Badajoz. La Serena, La Coronada, Río Zújar (MA-781349). Mérida, cerca del Guadiana (MA-139080). Baleares. Ibiza, Cala de Molins (BC-36082). Barcelona. San Vicets des Horts (MA-146890). Rubi (VAL-172200). Burgos. Pancorbo (139026). Hoyos del Tozo (MA-639895). Miranda del Ebro (MA-441679). Aranda del Duero (MA-139030). Cádiz. San Roque (MGC-55391). Algodonales (SEV-235033). Zahara de la Sierra, ró Guadalete (MA-460901). Cáceres. Madrigal de la Vera (MA-526055). Toril (SEV-103286). Puerto de Miravete (MA-139016). Alcántara (MA718802). Córdoba. Almodóvar del Río (MA-749268). Santa Eufemia (MA-773360). Cerca de Córdoba (BC-90513). Castellón. Arés de Maestrat (MA-628893). Nules (MA-139034). Segorbe (MA-139081). Ciudad Real. Arenas (MA-139009). El Osero (MA-139008). Cuenca. Montarco (MA-139041). Cañizares (MA-139089). 
Embalse de Alarcón (MA-530696). Gerona. Cabanas (MA-1390459). Figueras (MA- 139046). Granada. Pedro Martínez (MA-644565). Capileira (MA-214597). Albuñol (BC-840043). Sierra Nevada (MA-139079). Guadalajara. Zorita de los Canes (MA-194924). Roblelacasa (MA-483242). Fuensabiñán (MA-546965). Huesca. Javierre de Aro (SEV-235042). Presa de Lecina (BC-671598). Broto (BC-36072). Ascaso, Túnez de Balupar (MA-210939). Jaén. Sierra de Mágina (BC-36078). Mancha Real (MA-756757). Quesada (MA-480844). León. Garaño (MA-599552). Cacabelos (SALA-86395). Ponferrada, Margen del Sil (BC-78739). Lérida. Segría (BC-840048). Segría, Almatret (BC-840050). Cerca de Lérida (BC-36070). Logroño. Las Ruedas de Ocón (MA-589685). Grávalos (MA-533746). Autoñanzas (MA-351523). Lugo. Quiroga, Montefurado (SANT-31386). San Clodio, cerca del Sil (MA-139023). San Clodio, en cascajares del Sil (SANT-35194). Madrid. Cercedilla (MA-441685). Perales del Río (MA-440835). Cerro Negro (MA-139003). Málaga. Carratraca, sierra de Alcaparaín, (MA-139708). Sierra de la Pizarra (MGC-4886). Alhaurín el Grande (MGC-24818). Sierra de Almijara, Canillas de Aceituno, subida a la Maroma (MGC-41881). Murcia. Cartagena, Sierra de la Muela (MA-764065). Sierra Espuña (MA-139004). Caravaca (MA-409259). Lorca (BC-879516). Navarra. Fitero, Baños Viejos (MA-500042; BC-861760; MA-704607). Orense. Rubiá, Quereño, orilla del Sil (SANT-28106). Palencia. Cerca de Palencia MA-139374). Alar del Rey (MA-629910). Santibáñez de Ecla (MA-629911). Salamanca. Villaflores (BC-631199; MA-224626). Juzbado (MA-224625; BC-630390). Segovia. Brieva (MA-503136). Santiuste de Pedraza (MA-745751). Prádena (MA-488866). Cuéllar (MA-754336). Soria. Alconaba (MA-794215). Villar del Campo (MA-278781). Oluega (MA-139012). Ucero (MA-339558). Tarragona. Tortosa (BC-688084). Fredes (MA-626748). Cambrils (BC-879502). Puerto de Beceite (SALA-98510). Teruel. Villalquemado (MA-447160). Puerto de Alcalá de la Selva (MA-477306). Corbalán (MA-581257). Toledo. Iglesuela (MA-211970). El Arco, Calera (MA-173910). Navahermosa (MA-238521). Aranjuez (MA-441703). Valencia. Benicarló (MA-139084). Atzaneta d'Albaida (MA-446479). Buñol (MA-139035). Villar del Arzobispo (MA-346070). Valladolid. Encinas de Esgueva (MA-337936). Olmedo (MA-441677).
Portillo (MA-501814). Zamora. Almaraz del Duero (MA-202382). Toro (BC-93947). Cerca de Zamora (BC-91993). Zaragoza. Villamayor (MA-704606). Sierra de la Nava Alta (MA-484235). Calatayud (MA-139094).

PORTUGAL. Alto Alentejo. Elvas, cerca del río Guadiana (MA-180228); (MA-273055). Beira Baixa. Vila Velha de Rodao (SANT-26882, MA-139088 \& 273056). Tras os Montes. Bragança, Mogadouro, Bemposta (MA-823145). Barragem da Bemposta (MA-570390)

\section{A. agardhii}

ESPAÑA. Albacete. Nerpio, Sierra de las Cabras (MA-697075). Almería. Sierra María (MA-445716); (MA-139330); (BC-36105). Vélez Blanco, el Maimón (MGC-39654). Granada. Sierra Tejeda (MA-139326). La Puebla de Don Fadrique, La Sagra (MA-422234). Sierra de Baza (MA-139327). Jaén. Cazorla, Sierra de la Cabrilla (MA-480868). Cazorla, Sierra de Empanadas (MA-462219). Santiago de la Espada, Calar de las Palomas (MA-462220). Sierra de Mágina, El Serrate (MA-139337). Málaga. Sierra Tejeda (MA-701931); (BC-840040); (BC-36097); (BC-859494). Murcia. Moratalla, Revolcadores (MA-541907); (MA-238511); (MA-819786).

\section{Híbridos}

\section{Andryala integrifolia $\times$ A. arenaria}

ESPAÑA. Cádiz. Los Barrios (SEV-90303). Caños de Meca (SEV-234873). Córdoba. Rute (SEV-125999). Villanueva de Córdoba (SEV-147988). Cuenca. Villalba de la Sierra (VAL-149285). Huelva. Hinojos (SEV-126154). Villanueva de las Cruces (SEV-147988). Madrid. Villa del Prado, carretera al Encinar del Alberche (MA-566054). Málaga. Málaga, Finca de la Concepción (MGC-30643). Genalguacil (MGC-50839). Sevilla. Carmona (SEV-125643).

Toledo. Talavera de la Reina (MA-787365).

PORTUGAL. Ribatejo. Entre Porto Alto y Santo Estevao (SEV-286504). Beira Litoral. Cabo Mondego (SEV-235055). Coimbra, Arganil (MA-389489). Estremadura. Sines (MA-411711).

\section{Andryala integrifloia $\mathrm{x}$ A. rothia}

ESPAÑA. Cáceres. Guadalupe (MA-446604).

Cádiz. Vejer de la Frontera (MA-44076). Gibraltar (SEV-252559). Entre Villamartín y Prado del Rey 
(SEV-274185). Ciudad Real. Sierra Madrona (MA-596822). Córdoba. Hinojosa del Duque (SEV-23622b). Huelva. El Almendro (SEV-147997). Entre San Bartolomé de las Torres y Alosno (SEV-266489). Jaén. Aldeaquemada, Las Correderas (MA-224623). Madrid. Cadalso de los Vidrios, carretera del encinar del Alberche a Cadalso, junto al límite provincial con Toledo (MA-566056).

PORTUGAL. Tras os Montes. Freixo de Espada á Cinta, entre as barages de Alobadávila e de Saucelle (HVR-5354). Tejo Internacional (HVR-14152)

\section{Andryala integrifolia $\mathrm{x}$ A. ragusina}

ESPAÑA. Burgos. Obarenes (BC-879510). Pancorbo (MA-1319178). Valladolid, pinares (BC-879499). Segovia. Brieva (MA-503134), (MA-503135).

\section{Andryala arenaria $\mathrm{X}$ A. rothia}

ESPAÑA. Toledo. Quinta de Mora, Los Yébenes (MA-380430). Jaén. Santa Elena, Despeñaperros (BC-916138).

\section{Andryala cintrana $\times$ A integrifolia \\ PORTUGAL. Estremadura. Cascais, Cabo Raso (MA-807100).}

\section{Andryala cintrana $\times$ A. arenaria}

PORTUGAL. Estremadura. Ilhe Berlenga, devant le phare, Aout 1885, J. Daveau in Cl. Magnier, Flora Selecta Exsiccata n 1735 (MA-139297 a, b); idem, alrededores de la fortaleza, $31 \mathrm{~m}, 29^{\circ}$ $24^{\prime} 56^{\prime \prime} \mathrm{N}-9^{\circ} 30^{\prime} 23^{\prime \prime} \mathrm{W}, 20-\mathrm{VI}-2015$, D. Campos, C. Sánchez, M. Talavera \& S. Talavera, ${ }^{\circ}$ 180/15 (SEV-286494; idem, al N del restaurante, $62 \mathrm{~m}, 29^{\circ}$ 24'57' N - 9' 30'23' W, 20-VI-2015, D. Campos, C. Sánchez, M. Talavera \& S. Talavera, nº 183/15 (SEV-286945); idem, alrededores del faro de guía, 77 m, 39 24'54' N - 9 30'30' 'W, 20-VI-2015, D. Campos, C. Sánchez, M. Talavera \& S. Talavera, $n^{\circ} 192 / 15$ (SEV-286497) y no 194(4) SEV-286499). Cascais, Estrada do Guincho, cerca del faro de guía, a $30 \mathrm{~m}$ norte, arenales costeros, $10 \mathrm{~m}, 18$-VI-2015, D. Campos, C. Sánchez, M. Talavera \& S. Talavera, nº164/15 (SEV-286490).

AGRADECIMIENTOS. Agradecemos a los conservadores de los herbarios que nos enviaron los materiales que figuran en el texto, a Pilar FernándezPiedra y al Dr. Fco. Javier Salgeiro por la búsqueda bibliográfica, al Dr. Peter Gibbs por su ayuda de los textos en inglés, a Carlos Sánchez por la recolección de materiales de la Península Ibérica y Marruecos y al editor de esta serie en Acta Botanica Malacitana por sus comentarios y correcciones.

\section{BIBLIOGRAFÍA}

BURDET, H.M.,A. CHARPIN \& F. JACQUEMOUD -1983- Types nomenclaturaux des taxa ibériques décrits par Boissier ou Reuter. IV. Cistacées à Composées. Candollea 38: 751-802.

FEHRER, J., K. KRAK \& J. CHRTEK -2009Intra-individual polymorphism in diploid and apomictic polyploid hawkweeds (Hieracium, Lactuceae, Asteraceae): disentangling phylogenetic signal, reticulation, and noise. BMC Evolutionary Biology 9: 239.

GARCÍA ADÁ, R. -1992- Un híbrido nuevo en el género Andryala (Asteraceae). Acta Bot. Malacitana 17: 159-170

GARCÍAADÁ, R., G. LÓPEZ \& P. VARGAS -1996Notas botánicas sobre la flora vascular del centro de la Península Ibérica. Candollea 51: 373-380.

GREEN, M. L. -1929- The application of "nomina generica conservanda" to be determined by means of specified Standard-Species pp. 97-109, In: International Botanical Congress Cambridge (England), 1930. Nomenclature. Proposal by British Botanists (Prop. Brit. Bot). London.

HOUSE, P. -1993- In: C. E. JARVIS, F. R. BARRIE, D. M. ALLAN \& J. L. RAVEAL (eds.). A list of Linnaean generic names and their type. Reg. Veget. 127: 1-100 .

HOUSE, P. -1998-In: C. E. JARVIS \& N. TURLAND (eds.). Typification of Linnaean specific and varietal names in the Compositae (Asteraceae). Taxon 47: 347-370.

ROSELLÓ, A.J. \& L. SÁEZ -2000- Index Balearicum: An annotated check-list of the vascular plants described from the Balearic Islands. Collectanea Bot. 25: 3-203.

Dirección de los autores. Departamento de Biología Vegetal y Ecología (Botánica). Facultad de Biología, apartado 1095.41080-Sevilla.mtalavera@us.es 